\title{
Diol Mediated Tautomerization of Glycine: a DFT Study
}

\author{
Francis Sub*, Vanessa Rivera*, \& Ruben D. Parra \\ ${ }^{a}$ Department of Chemistry and Biochemistry, DePaul University, Chicago, IL 60614 \\ https:// doi.org/10.33697/ ajur.2019.014 \\ *Students: francisyjsub@gmail.com,vanessamarie124@gmail.com \\ Mentor:rparra1@depaul.edu
}

\begin{abstract}
The tautomerization of glycine via a triple proton transfer was investigated both in the gas phase and in aqueous solution using the B3LYP/6-31+G(d,p) level of theory. Fully optimized complexes of the neutral and zwitterion forms of glycine with 1,3propanediol were used for the reactant and product forms, respectively. The hydroxyl groups in the diol are conveniently oriented for glycine tautomerization through a concerted triple proton transfer facilitated by a network of three hydrogen bonds: $\mathrm{N}$ H...O-H...O-H...O=C. The activation energy for the zwitterion $\rightarrow$ neutral process increases in solution. Also, the diol-glycine complex favors the neutral over the zwitterion form in a vacuum, but the opposite is true in solution. For comparative purposes, the tautomerization of glycine via a three-proton transfer mediated by two molecules of water was also examined. The results are qualitatively similar, albeit with activation energies that are smaller to those found in the corresponding diol-mediated tautomerization.
\end{abstract}

\section{KEYWORDS}

Glycine; zwitterion, diol-mediated tautomerization; water-mediated tautomerization

\section{INTRODUCTION}

Amino acids occur predominantly in a neutral form in the gas phase and as a zwitterion in aqueous solution. ${ }^{1}$ The neutral and zwitterion forms are examples of constitutional isomers that differ in the location of a proton (i.e., tautomers). The interconversion between tautomers, also known as tautomerization, has been the subject of intense experimental and theoretical work. Being the simplest amino acid, glycine has been often used as a model system for tautomerization research. ${ }^{2-14}$ For example, theoretical calculations confirm the notion that the zwitterion form of glycine is not a minimum on the potential energy surface. However, it has also been found that two water molecules can make the zwitterion a local minimum. ${ }^{9}$ Most theoretical computations have focused on the direct intramolecular proton transfer step between the zwitterion and the neutral form, although some studies have examined the role of discrete water molecules to assist in the proton transfer. In particular, the waterassisted tautomerization in di-hydrated glycine has been reported. ${ }^{3,8}$ The notion of a water bridge to facilitate proton transfer has been applied as a convenient model to study proton transfer in biological proton pumps. ${ }^{15}$ Interestingly, polyols have also been found to facilitate long-range proton transfer. Specifically, polyols have been designed to possess a quasi-linear hydrogen-bonded network along their rigid and hydrophobic rod scaffold that facilitates transmembrane transport of protons. ${ }^{16,17}$ Moreover, polyols are also known to play a stabilizing role in proteins. ${ }^{18}$ To our knowledge, there has been neither theoretical nor experimental work on the proton transfer between glycine and a diol molecule. Because amino acids are the building blocks of peptides and hence proteins, this study is in part motivated to gain insight on the ability of a polyol to stabilize an amino acid in its neutral as well as in its zwitterion form. It is also of fundamental interest to examine the ability of the polyol to serve as a conduit for proton transfer leading to the transition from the zwitterion to the neutral amino acid. Given their relatively small sizes, glycine and 1,3propanediol were chosen as model systems for the calculations. It is worth noting that the 1,3-polyol system is a frequently occurring structural motif found in many biologically active natural products. ${ }^{19}$

In this work, the ability of a 1,3-propanediol to facilitate the tautomerization of glycine in the gas phase and the aqueous solution is investigated through density functional theory calculations. The results are compared with the equivalent tautomerization mediated by two water molecules. 


\section{COMPUTATIONAL METHODS}

All calculations were performed with the Gaussian 16 package. ${ }^{20}$ Structures were fully optimized with the B3LYP/6-31+G(d,p) method. Aqueous solution was modeled with the default method in Gaussian, i.e., SCRF= IEFPCM. Frequency calculations confirmed the nature of any optimized structure as either a minimum (all real frequencies) or a transition state (one imaginary frequency). Intrinsic reaction coordinate (IRC) calculations were performed to confirm that the transition states connect the intended minima. Complexation energies were corrected for basis set superposition error (BSSE) using the counterpoise method. $^{21}$

\section{RESULTS AND DISCUSSION}

The optimized structures for the diol-mediated glycine tautomerization in the gas phase are illustrated in Figure 1. The appearances of the resulting geometries in solution are similar to their gas phase counterparts and hence are not shown. Inspection of Figure 1 reveals that the glycine-diol dimers are stabilized primarily by a sequence of three hydrogen bonds involving the two $\mathrm{OH}$ groups in the diol, the nitrogen and one of the oxygen atoms in glycine. Interestingly, the conformation adopted by glycine in the dimers is such that the oxygen atom in glycine farther from the nitrogen center is the one participating in the $\mathrm{H}$ bond. Previous theoretical and experimental work has consistently demonstrated that the zwitterion does not exist in the gas phase. However, the three $\mathrm{H}$-bonds $\mathrm{N}_{8}-\mathrm{H}_{3} \cdots \mathrm{O}_{16}-\mathrm{H}_{14} \cdots \mathrm{O}_{15}-\mathrm{H}_{13} \cdots \mathrm{O}_{2}=\mathrm{C}_{1}$ in the dimer appear to be strong enough to stabilize the glycine in its zwitterion form. Indeed, the relatively short H-bond distances (Table 1) and quasilinear angles (Table 2) confirm the presence of strong $\mathrm{H}$ bonds.

Moreover, the zwitterion is stabilized by an intramolecular $\mathrm{H}$ bond, $\mathrm{N}_{8}-\mathrm{H}_{10} \cdots \mathrm{O}_{4}=\mathrm{C}_{1}$, with the closer carboxylate oxygen. The calculated BSSE-corrected interaction energy for the zwitterion-diol dimer $(\Delta \mathrm{E}=-25.13 \mathrm{kcal} / \mathrm{mol})$ demonstrates the strength of the $\mathrm{H}$ bond interactions responsible for the stabilization of the zwitterion in the gas phase. A triple proton transfer leads to the dimer of neutral glycine and the diol molecule, neutral-diol dimer, as shown in Figure 1. H bond distances listed in Table 1 suggest somewhat weaker $\mathrm{H}$ bonds than found in the zwitterion-diol dimer. For example, the intramolecular $\mathrm{N}-\mathrm{H} \cdots \mathrm{O}=\mathrm{C}$ appears weaker in the neutral-diol dimer, with an $\mathrm{H}$ bond distance $(2.457 \AA)$ much longer than that in the zwitterion-diol dimer $(1.737 \AA)$. The weaker $\mathrm{H}$ bond strengths are confirmed by the smaller magnitude of the calculated BSSE-corrected interaction energy for the neutral-diol dimer $(\Delta \mathrm{E}=-19.28 \mathrm{kcal} / \mathrm{mol})$. Although geometry optimization in aqueous solution does not change the overall appearances of the dimers, it does bring about some noticeable changes in the $\mathrm{H}$ bond distances. Specifically, the zwitterion-diol dimer in solution exhibits longer $\mathrm{H}$ bond distances, except for the $\mathrm{O}_{15}-\mathrm{H}_{13} \cdots \mathrm{O}_{2}=\mathrm{C}_{1}$. In contrast, the neutral-diol dimer in solution exhibits shorter $\mathrm{H}$ bond distances. The intramolecular $\mathrm{N}_{8}-\mathrm{H}_{10} \cdots \mathrm{O}_{4}=\mathrm{C}_{1}$ bond in aqueous solution is elongated to $2.151 \AA$ and 2.781 $\AA$ in the zwitterion-diol and neutral-diol dimers, respectively.
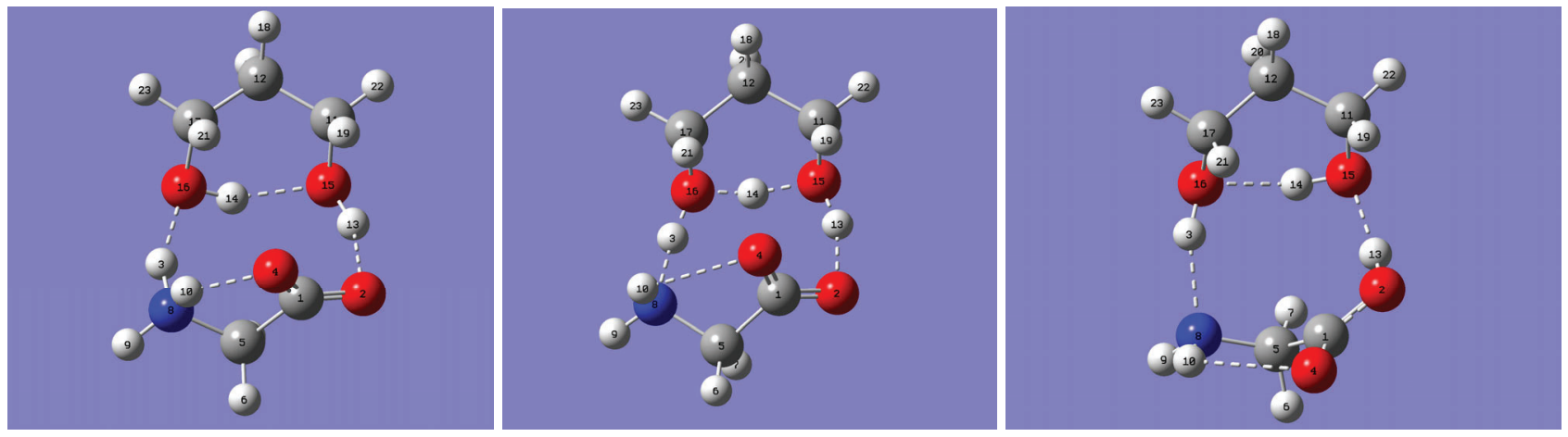

Figure 1. B3LYP/6-31+G(d,p) optimized geometries for the zwitterion-diol (left), transition state (middle), and neutral-diol (right) complexes in the gas phase.

Diol Complexes (Gas Phase)

\begin{tabular}{ccccccc}
\hline Complex & $\mathbf{N}_{8}-\mathbf{H}_{3}$ & $\mathbf{H}_{3} \cdots \mathbf{O}_{16}$ & $\mathbf{O}_{16}-\mathbf{H}_{14}$ & $\mathbf{H}_{14} \cdots \mathbf{O}_{15}$ & $\mathbf{O}_{15}-\mathbf{H}_{13}$ & $\mathbf{H}_{13} \cdots \mathbf{O}_{2}$ \\
\hline Zwitterion & 1.053 & 1.743 & 0.987 & 1.782 & 0.984 & 1.844 \\
Neutral & 1.866 & 0.990 & 1.816 & 0.982 & 1.729 & 0.997 \\
TS & 1.492 & 1.092 & 1.157 & 1.278 & 1.038 & 1.506
\end{tabular}


Diol Complexes (Aqueous Solution)

\begin{tabular}{ccccccc}
\hline Complex & $\mathbf{N}_{8}-\mathbf{H}_{3}$ & $\mathbf{H}_{3} \cdots \mathbf{O}_{16}$ & $\mathbf{O}_{16}-\mathbf{H}_{14}$ & $\mathbf{H}_{14} \cdots \mathbf{O}_{15}$ & $\mathbf{O}_{15}-\mathbf{H}_{13}$ & $\mathbf{H}_{13} \cdots \mathbf{O}_{2}$ \\
\hline Zwitterion & 1.049 & 1.789 & 0.982 & 1.828 & 0.984 & 1.820 \\
Neutral & 1.809 & 0.996 & 1.796 & 0.985 & 1.650 & 1.007 \\
TS & 1.501 & 1.080 & 1.217 & 1.200 & 1.067 & 1.415
\end{tabular}

Water Complexes (Gas Phase)

\begin{tabular}{ccccccc}
\hline Complex & $\mathbf{N}_{8}-\mathbf{H}_{3}$ & $\mathbf{H}_{3} \cdots \mathbf{O}_{16}$ & $\mathbf{O}_{16}-\mathbf{H}_{14}$ & $\mathbf{H}_{14} \cdots \mathbf{O}_{15}$ & $\mathbf{O}_{15}-\mathbf{H}_{13}$ & $\mathbf{H}_{13} \cdots \mathbf{O}_{2}$ \\
\hline Zwitterion & 1.046 & 1.771 & 0.993 & 1.735 & 0.997 & 1.716 \\
Neutral & 1.811 & 0.998 & 1.755 & 0.990 & 1.731 & 0.993 \\
TS & 1.200 & 1.318 & 1.175 & 1.252 & 1.226 & 1.193
\end{tabular}

Water Complexes (Aqueous Solution)

\begin{tabular}{ccccccc}
\hline Complex & $\mathbf{N}_{8}-\mathbf{H}_{3}$ & $\mathbf{H}_{3} \cdots \mathbf{O}_{16}$ & $\mathbf{O}_{16}-\mathbf{H}_{14}$ & $\mathbf{H}_{14} \cdots \mathbf{O}_{15}$ & $\mathbf{O}_{15}-\mathbf{H}_{13}$ & $\mathbf{H}_{13} \cdots \mathbf{O}_{2}$ \\
\hline Zwitterion & 1.047 & 1.774 & 0.981 & 1.775 & 0.990 & 1.770 \\
Neutral & 1.787 & 1.002 & 1.727 & 0.993 & 1.632 & 1.006 \\
TS & 1.420 & 1.122 & 1.205 & 1.213 & 1.105 & 1.339
\end{tabular}

Table 1. Relevant bond distances $(\AA)$ for the glycine-diol and glycine-(water) 2 complexes in the gas phase and solution.

Optimized geometries of each of the glycine tautomers with two water molecules in the gas phase are shown in Figure 2. It should be noted that the optimized complexes of glycine with the two molecules of water resemble closely those reported previously by other researchers using DFT and reactive force field molecular dynamics simulations (ReaxFF MD). As with the glycine-diol dimers, the glycine-(water) 2 geometries in aqueous solutions are very similar to their gas phase counterparts.

Inspection of Figures 1 and 2 reveals the striking similarity of the diol-glycine dimers and the corresponding glycine-(water) $)_{2}$ complexes. Moreover, an inspection of Table 1 and $\mathbf{2}$ shows that the structural changes in solution relative to the gas phase in these complexes are qualitatively similar to those previously noted in the glycine-diol dimers. The structural similarities of the dimers in the gas phase can be further corroborated by the BSSE-interaction energies of the zwitterion- $(\text { water })_{2}(-27.65 \mathrm{kcal} / \mathrm{mol})$ and the neutral-(water) $2(-21.89 \mathrm{kcal} / \mathrm{mol})$ which compare fairly well with those of the zwitterion-diol $(-25.13 \mathrm{kcal} / \mathrm{mol})$ and the neutral-diol $(-19.28 \mathrm{kcal} / \mathrm{mol})$ discussed before. It is important to note that the two molecules of water in the complexes were effectively considered as one unit to calculate interaction energies. Thus, the two water molecules in the complex were taken as one unit, and the glycine (zwitterion or neutral) as the other unit.
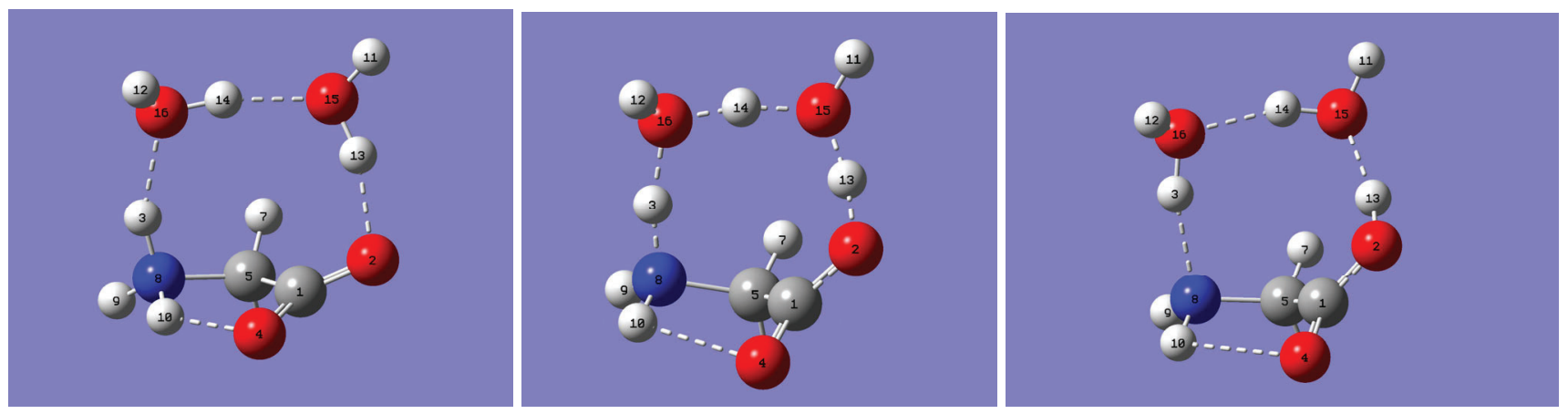

Figure 2. B3LYP/6-31+G(d,p) optimized geometries for the zwitterion-(water) 2 (left), transition state (middle), and neutral-(water) 2 (right) complexes in the gas phase. 
Diol Complexes (Gas Phase)

\begin{tabular}{ccccc}
\hline Complex & $\mathbf{N}_{8}-\mathbf{H}_{3}-\mathbf{O}_{16}$ & $\mathbf{H}_{3}-\mathbf{O}_{16} \cdots \mathbf{H}_{15}-\mathbf{O}_{15}$ & $\mathbf{O}_{15}-\mathbf{H}_{3} \cdots \mathbf{O}_{2}=\mathbf{C}_{1}$ & $\mathbf{O}_{2}=\mathbf{C}_{1}-\mathbf{C}_{5}-\mathbf{N}_{8}$ \\
\hline Zwitterion & 156.8 & 150.3 & 156.2 & -154.2 \\
Neutral & 158.2 & 147.9 & 159.9 & -126.5 \\
TS & 171.7 & 161.4 & 159.8 & -131.6
\end{tabular}

Diol Complexes (Aqueous Solution)

\begin{tabular}{ccccc}
\hline Complex & $\mathbf{N}_{8}-\mathbf{H}_{3}-\mathbf{O}_{16}$ & $\mathbf{H}_{3}-\mathbf{O}_{16} \cdots \mathbf{H}_{15}-\mathbf{O}_{15}$ & $\mathbf{O}_{15}-\mathbf{H}_{3} \cdots \mathbf{O}_{2}=\mathbf{C}_{1}$ & $\mathbf{O}_{2}=\mathbf{C}_{1}-\mathbf{C}_{5}-\mathbf{N}_{8}$ \\
\hline Zwitterion & 159.5 & 149.3 & 164.0 & -146.7 \\
Neutral & 163.0 & 148.6 & 165.8 & -104.2 \\
TS & 167.2 & 162.2 & 170.9 & -110.0
\end{tabular}

Water Complexes (Gas Phase)

\begin{tabular}{ccccc}
\hline Complex & $\mathbf{N}_{8}-\mathbf{H}_{3}-\mathbf{O}_{16}$ & $\mathbf{H}_{3}-\mathbf{O}_{16} \cdots \mathbf{H}_{15}-\mathbf{O}_{15}$ & $\mathbf{O}_{15}-\mathbf{H}_{3} \cdots \mathbf{O}_{2}=\mathbf{C}_{1}$ & $\mathbf{O}_{2}=\mathbf{C}_{1}-\mathbf{C}_{5}-\mathbf{N}_{8}$ \\
\hline Zwitterion & 156.1 & 167.5 & 163.4 & -151.3 \\
Neutral & 163.5 & 161.5 & 161.5 & -123.7 \\
TS & 166.0 & 167.7 & 169.3 & -121.5
\end{tabular}

Water Complexes (Aqueous Solution)

\begin{tabular}{ccccc}
\hline Complex & $\mathbf{N}_{8}-\mathbf{H}_{3}-\mathbf{O}_{16}$ & $\mathbf{H}_{3}-\mathbf{O}_{16} \cdots \mathbf{H}_{15}-\mathbf{O}_{15}$ & $\mathbf{O}_{15}-\mathbf{H}_{3} \cdots \mathbf{O}_{2}=\mathbf{C}_{1}$ & $\mathbf{O}_{2}=\mathbf{C}_{1}-\mathbf{C}_{5}-\mathbf{N}_{8}$ \\
\hline Zwitterion & 159.1 & 168.1 & 166.6 & -147.0 \\
Neutral & 167.2 & 161.4 & 173.0 & -96.4 \\
TS & 170.5 & 169.0 & 173.7 & -99.8
\end{tabular}

Table 2. Relevant bond angles (degrees) for the glycine-diol and glycine-(water) 2 complexes in the gas phase and solution.

As shown in Figures 1 and 2, the zwitterion-neutral tautomerization assisted by 1-3-propanediol or two molecules of water proceeds through a concerted triple proton transfer reaction. The relative electronic energies with zero-point energy corrections, $\Delta \mathrm{E}_{\mathrm{o}}$, are listed in Table 3 for both the gas phase and the aqueous solution reactions. In the gas phase, the complexes containing the neutral tautomer are favored. In solution, however, the zwitterion-containing complexes are energetically more stable. The larger stability of the zwitterion complexes can be traced down to stronger solute-solvent stabilizing interactions. Although the zwitterion carries no net charge, it is, however, a highly polar molecule. Indeed, it is found that in the gas phase the dipole moment of the zwitterion-diol complex, for example, is almost twice that of the neutral-diol complex (6.46 D vs $3.41 \mathrm{D})$, with similar results for the glycine-(water) $)_{2}$ complexes. The large difference in dipole moments is in line with the larger charge separation in the zwitterion, and it also suggests that in a polar solvent, the zwitterion-complex would be stabilized more than the neutral complex counterpart. Reaction enthalpies, $\Delta \mathrm{H}$, and reaction free energies, $\Delta \mathrm{G}$, listed in Table 3 are relatively close to each other and with $\Delta \mathrm{E}_{\circ}$. Also listed in Table 3 are the corresponding activation energies. Relative to the gas phase, the activation energies in solution are found to be consistently higher. Thus, the solvent stabilization of the zwitterion-complex appears to be larger than the corresponding stabilization of the transition-state complex (in either the diol- or water-assisted tautomerization). The result appears consistent with the larger dipole moment of the zwitterion-complex when compared with that of the pertinent transition state. For example, the dipole moment for the transition state in the diol-complex is $5.75 \mathrm{D}$, somewhat smaller than the zwitterion-diol complex $(6.46 \mathrm{D})$. In addition to the difference in dipole moments, some structural changes occur for the transition state geometries that may in part contribute to the noted increase in activation energy upon solvation. In particular, the intramolecular $\mathrm{N}-\mathrm{H} \cdots \mathrm{O}=\mathrm{C}$ interaction appears to weaken upon solvation. Specifically, the intramolecular $\mathrm{N}_{8}-\mathrm{H}_{10} \cdots \mathrm{O}_{4}=\mathrm{C}_{1}$ bond in aqueous solution is elongated to $2.657 \AA$ from the gas phase value of $2.386 \AA$ in the transition state of the diol-containing complexes. This change is also reflected on the 20 degrees change in the $\mathrm{O}_{2}=\mathrm{C}_{1}-\mathrm{C}_{5}-\mathrm{N}_{8}$ dihedral angle (see Table 2). Likewise, the intramolecular $\mathrm{N}_{8}-\mathrm{H}_{10} \cdots \mathrm{O}_{4}=\mathrm{C}_{1}$ bond in aqueous solution is elongated to $2.803 \AA$ from the gas phase value of $2.343 \AA$ in the transition state of the water-containing complexes. 
Diol-Assisted Tautomerization

\begin{tabular}{lcccccc}
\hline Medium & $\boldsymbol{\Delta} \mathbf{E o}$ & $\boldsymbol{\Delta} \mathbf{H}$ & $\boldsymbol{\Delta} \mathbf{G}$ & $\boldsymbol{\Delta} \mathbf{E \mathbf { o } ^ { \ddagger }}$ & $\boldsymbol{\Delta} \mathbf{H}^{\ddagger}$ & $\boldsymbol{\Delta} \mathbf{G}^{\ddagger}$ \\
\hline Gas & -5.90 & -5.79 & -5.99 & 5.21 & 4.46 & 6.51 \\
Phase & 3.29 & 3.17 & 3.60 & 8.35 & 7.51 & 9.57 \\
Aqueous & & & & & &
\end{tabular}

Water-Assisted Tautomerization

\begin{tabular}{lcccccc}
\hline Medium & $\boldsymbol{\Delta E \mathbf { E }}$ & $\boldsymbol{\Delta} \mathbf{H}$ & $\boldsymbol{\Delta} \mathbf{G}$ & $\boldsymbol{\Delta} \mathbf{E o}^{\ddagger}$ & $\Delta \mathbf{H}^{\ddagger}$ & $\Delta \mathbf{G}^{\ddagger}$ \\
\hline Gas & -7.12 & -7.05 & -7.27 & 3.62 & 2.53 & 4.94 \\
Phase & 2.44 & 2.26 & 2.59 & 7.49 & 6.31 & 8.75 \\
Aqueous & & & & &
\end{tabular}

Table 3. Reaction electronic energies with zero-point corrections $\left(\Delta \mathrm{E}_{0}\right)$, enthalpies $(\Delta \mathrm{H})$ and free energies $(\Delta \mathrm{G})$ at $298 \mathrm{~K}$ and corresponding activation energies for the glycine tautomerization reactions. Values in $\mathrm{kcal} / \mathrm{mol}$.

Although no previous work has been reported on diol-assisted tautomerization of glycine, the results presented here agree, at least qualitatively, with previous reports that show the neutral form of glycine to be the dominant species in the gas phase, while the zwitterion form to be the preferred one in solution. For example, titration studies have suggested that the free energy of the zwitterion is $7.3 \mathrm{kcal} / \mathrm{mol}$ lower than its neutral counterpart in an aqueous solution. ${ }^{14}$ Moreover, the zwitterion has been reported to be unstable in the gas phase and to transform itself into the neutral form without any energy barrier. Some gas phase calculations suggest that the complex of zwitterion with two discrete water molecules is still higher in energy $(\Delta \mathrm{E}=11.3 \mathrm{kcal} / \mathrm{mol}$ at the MP2//HF/6-31++G(d,p) level, and without zero-point energy corrections) than the corresponding most stable complex of the neutral tautomer. ${ }^{10}$ The relative order is reversed upon inclusion of solvent effects through the Polarized Continuum Model, PCM, with the zwitterion complex lying $4.4 \mathrm{kcal} / \mathrm{mol}$ below the neutral complex. It should be noted that the zwitterion complex with two water molecules has essentially the same geometrical orientation as the one considered here. However, the most stable neutral complex (reference 10) has the two water molecules forming a ring with the carboxylic acid end of glycine.

In addition to relative energies, the free energy of activation has also been extensively studied. For example, experimental results indicate that the zwitterion-neutral tautomerization in glycine has a free energy of activation of $14.4 \mathrm{kcal} / \mathrm{mol}{ }^{22} \mathrm{Computational}$ studies provide different activation energies depending on the methods used or the mechanistic approach employed. An activation free energy of $5.42 \mathrm{kcal} / \mathrm{mol}$ has been reported for a direct intramolecular zwitterion-neutral proton transfer using B3LYP/6-31++G(d,p) and the IEFPCM model to account for solvent (aqueous) effects. ${ }^{6}$ Molecular dynamics simulations, on the other hand, have yielded activation energies of about $11.6 \mathrm{kcal} / \mathrm{mol},{ }^{5} 12.7 \mathrm{kcal} / \mathrm{mol},{ }^{7}$ and about $17.0 \mathrm{kcal} / \mathrm{mol} ., 14$ An assisted triple proton transfer mechanism in aqueous solution considering two molecules of water has also been investigated. An activation energy of $15.8 \mathrm{kcal} / \mathrm{mol}$ has been estimated through molecular dynamics simulations using a reactive force field developed to describe proton transfer in glycine. ${ }^{3}$

\section{CONCLUSIONS}

To the best of the authors' knowledge, the first study on the diol-assisted tautomerization of glycine is reported in this study. Calculations were performed at the B3LYP/6-31+G(d,p) level of theory in the gas phase and aqueous solution using the IEFPCM method. Dimer complexes between 1,3-propanediol and glycine (zwitterion and neutral form) were chosen as model systems. Strong hydrogen bonds stabilize the complexes, especially the complex of the zwitterion form of glycine. The mechanistic pathway investigated consisted of a triple proton transfer facilitated by the two OH groups from the diol acting as a bridge between the carboxyl and the amine group. The same mechanistic pathway was examined considering two molecules of water instead of the diol molecule. The results for both diol-mediated and water-mediated tautomerization appear comparable to one another and in qualitative agreement with other relevant experimental and theoretical studies. The results of this study should serve as motivation for exploring further the role that a diol, and in general a polyol, may play in tautomerization and other proton transfer reactions. Additional studies need to be conducted to address some of the limitations inherent to this study. For example, the results presented in this work refer to the static geometries of the optimized complexes, and it should be therefore a subject of future work to examine the dynamics of the interactions, for example by using ab initio molecular dynamics simulations. It is also desirable to consider other density functional methods that can better account for dispersion corrections, such as the APFD functional. Alternatively, the use of correlated methods such as MP2 or CCSD should also be explored along with correlationconsistent basis sets. Because of the impractical cost of computational methods that can account for both static and dynamic 
electron correlation, a good compromise between computational cost and accurate computation of thermochemical quantities is usually offered by composite methods such as the CBS-QB3 method, and therefore future studies should consider such composite method to improve upon the predicted results presented in this work. Although the default method for modeling chemistry in solution in the Gaussian 16 package was used in this study, it is nonetheless desirable that other approaches be considered. For example, the SDM method has been found to perform well for predicting reaction energies in solution. ${ }^{23}$ Future studies should also consider explicitly the conformational space of both the diol and glycine/zwitterion to account for the energy cost, if any, in adopting the particular conformations adopted by the molecules in the reactions both in the gas phase and in solution. Although the concerted nature of the multiple-proton transfer was noted in this study, additional studies need to be carried out to determine whether the transfer is synchronous or asynchronous. For this purpose, obtaining the reaction force and force constant profiles along the intrinsic reaction coordinate, or IRC would prove useful. ${ }^{24}$ Furthermore, although no intermediate species was found in the transition from zwitterion-complex to neutral-complex rendering the reaction to be concerted, it is still subject for future work whether this remains so upon using higher levels of theory. Presumably, intermediates species would need to be stabilized enough to be detected computationally. It might also be possible that increasing the polyol chain would give rise to intermediate species. Indeed, it has been reported that a single charge center in polyol chains can be significantly stabilized by the hydrogen-bonded network, and it can also enhance the interaction energy between adjacent noncharged $\mathrm{OH}$ groups in the network. ${ }^{25}$ The results suggest the intriguing possibility that a sequential rather than a concerted proton transfer may occur under these conditions. Thus, using higher polyol chains, namely a triol or tetraol to assist glycine (and other amino acids) tautomerization, will be considered in our group and the results published elsewhere.

\section{ACKNOWLEDGMENTS}

The authors thank the Department of Chemistry and Biochemistry of DePaul University for support.

\section{REFERENCES}

1. Schmidt, J., and Kass, S. R. (2013) Zwitterion vs Neutral Structures of Amino Acids Stabilized by a Negatively Charged Site: Infrared Photodissociation and Computations of Proline-Chloride Anion, J. Phys. Chem. A 117, 4863-4869. https://pubs.acs.org/doi/abs/10.1021/jp402267c

2. Kim, C. K., Park, B-H., Lee, H. W., and Kim, C. K. (2013) Comprehensive Studies on the Tautomerization of Glycine: A Theoretical Study, Org. Biomol. Chem. 11 14007-1413. bttps://pubs.rsc.org/en/content/articlelanding/2013/ob/c2ob26602d

3. Rahaman, O., van Duin, A. C. T., Goddard III, W. A., and Doren, D. J. (2011) Development of a ReaxFF Reactive Force Field for Glycine and Application to Solvent Effect and Tautomerization, J. Phys. Chem. B. 115, $249-261$. https://pubs.acs.org/doi/abs/10.1021/jp108642r

4. Tang, W., Cai, C., Zhao, S., and Liu, H. (2018) Development of Reaction Density Functional Theory and Its Application to Glycine Tautomerization Reaction in Aqueous Solution, J. Phys. Chem. C. 122, 20745-20754. bttps://pubs.acs.org/doi/abs/10.1021/acs.jpcc.8605383

5. Tolosa, S., Hidalgo, A., and Sansón, J. A. (2012) Amino Acid Tautomerization Reactions in Aqueous Solution via Concerted and Assisted Mechanisms Using Free Energy Curves from MD Simulation, J. Phys. Chem. B. 116, 13033-13044. https://pubs.acs.org/doi/abs/10.1021/jp307391s

6. Tuñon, I., Silla, E., and Ruiz-Lopéz, M. F. (2000) On the Tautomerization Process of Glycine in Aqueous Solution, Chem. Phys. Lett. 321, 433-437. bttps://doi.org/10.1016/S0009-2614(00)00365-1

7. Leung, K., and Rempe, B. S. (2005) Ab Initio Molecular Dynamics Study of Glycine Intramolecular Proton Transfer in Water, J. Chem. Phys. 122, 184506(1)-184506(12). https:/ / aip.scitation.org/doi/10.1063/1.1885445

8. Balta, B., and Aviyente, V. (2004) Solvent Effects on Glycine II. Water-Assisted Tautomerization, J. Comp. Chem. 25, 690-703. https://doi.org/10.1002/jcc.10422

9. Jensen, J. H., and Gordon, M. S. (1995) On the Number of Water Molecules Necessary to Stabilize the Glycine Zwitterion, J. Am. Chem. Soc. 117, 8159-8170. https://pubs.acs.org/doi/abs/10.1021/ja00136a013

10. Aikens C. M., and Gordon, M. S. (2006) Incremental Solvation of Nonionized and Zwitterionic Glycine, J. Am. Chem. Soc. 128, 12835-12850. https://pubs.acs.org/doi/10.1021/ja062842p

11. Fernández-Ramos, A., Smedarchina, Z., Siebrand, W., and Zgierski, M. Z. (2000) A Direct-Dynamics Study of the Zwitterionto-Neutral Interconversion of Glycine in Aqueous Solution, J. Chem. Phys. 113, 9714-9721. https://doi.org/10.1063/1.1322084

12. Pérez de Tudela, R., and Marx, D. (2016) Water-Induced Zwitterionization of Glycine:Stabilization Mechanism and Spectral Signatures, J. Phys. Chem. Lett 7, 5137-5142. https://pubs.acs.org/doi/full/10.1021/acs.jpclett.6b02247

13. Wada, G., Tamura, E., Okina, M., and Nakamura, M.(1982) On the Ratio of Zwitterion Form to Uncharged Form of Glycine at Equilibrium in Various Aqueous Media, Bull. Chem, Soc. Jpn. 55, 3064-3067. bttps:// doi.org/10.1246/bcsj.55.3064

14. Okuyama-Yoshida, N., Nagaoka, M., and Yamabe, T. (1998) Origin of the Transition State on the Free Energy Surface: Intramolecular Proton Transfer Reaction of Glycine in Aqueous Solution, J. Phys. Chem. 102, 285-292.

https://pubs.acs.org/doi/abs/10.1021/jp982534g 
15. Konig, P. H., Ghosh, N., Hoffmann, M., Elstner, M., Tajkhorshid, E., Frauenheim, Th., and Cui, Q. (2006) Toward theoretical analysis of long-range proton transfer kinetics in biomolecular pumps, J. Phys. Chem. A 110, 548-563. bttps://pubs.acs.org/doi/10.1021/jp052328q

16. Weiss, L. A., Sakai, N., Ghebremariam, B., Ni, C., and Matile S. (1997) Rigid Rod-Shaped Polyols: Functional Nonpeptide Models for Transmembrane Proton Channels J. Am. Chem. Soc. 119, 12142-12149. bttss:/ / pubs.acs.org/doi/abs/10.1021/ja973126d

17. Sakai, N., Mareda, J., and Matile S. (2005) Rigid-Rod Molecules in Biomembrane Models: From Hydrogen-Bonded Chains to Synthetic Multifunctional Pores. Acc. Chem. Res. 38, 79-87. https://pubs.acs.org/doi/abs/10.1021/ar0400802

18. Gekko, K. (1981) Enthalpy and entropy of transfer of amino acids and diglycine from water to aqueous polyol solutions $J$. Biochem. 90, 1643-1652. https:// doi.org/10.1093/oxfordjournals.jbchem.a133639

19. Iwata, M., Yazaki, R., Suzuki, Y., Kumagai, N., and Shibasaki, M. (2009) Direct catalytic asymmetric aldol reactions of thioamides: Toward a stereocontrolled synthesis of 1,3-Polyols. J. Am. Chem. Soc. 131, 18244-18245. bttps://pubs.acs.org/doi/10.1021/ja909758e

20. Frisch, M. J., Trucks, G.W., Schlegel, H.B., et al. (2016) Gaussian 16, revision B.01. Gaussian, Inc.: Wallingford, CT. https://gaussian.com/citation/

21. Boys, S. F., and Bernardi, F. (1970) The Calculation of Small Molecular Interactions by the Differences of Separate Total Energies. Some Procedures with Reduced Errors, Mol. Phys. 19, 553-566. bttps:/ / www.tandfonline.com/doi/abs/10.1080/00268977000101561

22. Slifkin, M.A., and Ali, S.M. (1984) Thermodynamic Parameters of the Activation of Glycine Zwitterion Protonation Reactions, J. Mol. Liq. 4, 215-221. https:/ / doi.org/10.1016/0167-7322(84)80025-2

23. Marenich, A. V., Cramer, C. J., and Truhlar, D. G. (2009) Universal-solvation model based on solute electron density and on a continuum model of the solvent defined by the bulk dielectric constant and atomic surface tensions, J. Phys. Chem. B, 113, 6378-6396. https://pubs.acs.org/doi/abs/10.1021/jp810292n

24. Yepes, J. S. Murray, P. Politzer, P. Jaque, (2012) The reaction force constant: an indicator of the synchronicity in double proton transfer reactions, Phys. Chem. Chem. Phys. 14, 11125-11134. https:/ / pubs.rsc.org/en/ content/ articlelanding/2012/cp/c2cp41064h

25. Shokri, A., Schmidt, J., Wang, X-B., and Kass, S. R. (2012) Hydrogen Bonded Arrays: The power of multiple hydrogen bonds, J. Am. Chem. Soc. 134, 2094-2099. https://pubs.acs.org/doi/abs/10.1021/ja2081907

\section{ABOUT STUDENT AUTHORS}

Francis Suh will graduate in 2019 with a Bachelors of Science degree in Chemistry and intends to further her studies by pursuing a Ph.D. in chemistry. She plans to start her Ph.D. program in Organic Chemistry in the fall of 2019, at the University of Illinois - at Chicago. Vanessa Rivera graduated in 2018 with a Bachelors of Science degree in Chemistry and is currently obtaining a Doctorate in Pharmacy at Rosalind Franklin University of Medicine and Science.

\section{PRESS SUMMARY}

To our knowledge, there has been neither theoretical nor experimental work on the proton transfer between glycine and a diol molecule. The tautomerization of glycine via a triple proton transfer was investigated both in the gas phase and in aqueous solution using computational studies. It has been shown that the hydrogen bond from the glycine facilitates the proton transfer between glycine and a diol molecule. It was also shown for the diol-glycine complex to favor the neutral over the zwitterion form in a vacuum, but the opposite is true in solution. 